\title{
Motion of a Colloidal Particle Coated with a Layer of Adsorbed Polymers in a Spherical Cavity
}

\author{
HuAN J. KEH ${ }^{1}$ AND JiMMY KuO \\ Department of Chemical Engineering, National Taiwan University, Taipei 106-17 Taiwan, Republic of China
}

Received April 15, 1996; accepted September 9, 1996

\begin{abstract}
An analytical study is presented for the quasisteady translation and steady rotation of a spherical particle covered by a layer of adsorbed polymers located at the center of a spherical cavity that may also have an adsorbed polymer layer on its inside wall. The Reynolds number is assumed to be small, and the surface polymer layers are assumed to be thin with respect to the particle radius and the spacing between solid surfaces. To solve the Stokes flow equations within and outside the polymer layers a method of matched asymptotic expansions in small parameters $\lambda_{1}$ and $\lambda_{2}$ is used, where $\lambda_{1}$ and $\lambda_{2}$ are the ratios of the polymer-layer length scale to the radius of curvature at the particle surface and at the cavity wall, respectively. The results for the hydrodynamic force and torque exerted on the particle are expressed as an effective hydrodynamic thickness $(L)$ of the adsorbed polymer layer surrounding the particle, which are accurate to $O\left(\lambda_{1}^{2}\right)$. The $O\left(\lambda_{1}\right)$ term for $L$ normalized by its value in the absence of the cavity is found to be independent of the polymer segment distribution, the hydrodynamic interactions among the segments, and the volume fraction of the segments. The $O\left(\lambda_{1}^{2}\right)$ term for $L$, however, is a sensitive function of the polymer segment distribution and the volume fraction of the segments. In general, the boundary effects on the motion of a polymer-coated particle can be quite significant in appropriate situations. $\odot 1997$ Academic Press
\end{abstract}

Key Words: particle translation; particle rotation; adsorbed polymers; hydrodynamic thickness; boundary effects.

\section{INTRODUCTION}

The adsorption of polymers at solid-liquid interfaces is of practical interest in various fields and has been a subject of many theoretical (1-6) and experimental (7-12) investigations. Coating of colloidal particles with polymers plays an important role in the control of the stability/flocculation behavior of colloidal suspensions (13). The interactions between polymer and particle generate nonuniform distributions of polymer throughout the solution and influence the energy between particles (14). Another spectacular effect of such adsorption is the restriction of flow in capillaries due

\footnotetext{
${ }^{1}$ To whom correspondence should be addressed.
}

to the effective decrease in size (15). The attachment of polymers to microporous membranes offers the possibility of manipulating the transport rate of solvent and solutes and negating the adverse effects of pore-size distribution on membrane separations (16).

The structure of an adsorbed polymer layer depends on the nature of the polymer, the solvent, and the interface. In general, the adsorbed polymer chains consist of a collection of "trains," in which each polymer segment contacts the interface, "loops,' in which only the initial and final segments attach to the interface, and "tails," which begin at the interface but terminate in the solution $(17,18)$. Since the adsorbed polymer layer is diffuse, there is no unique measure of its thickness. One convenient definition, applicable to both colloidal particles and micropores, is the hydrodynamic thickness which is the distance the "no-slip" boundary condition on the fluid velocity must be moved into the fluid phase to produce the same hydrodynamic effect as the polymer layer. For the case of a polymer layer that is thin relative to the radii of curvature of the solid surface, previous theoretical analyses (19-21) predict the same value of the hydrodynamic thickness for different external flows and geometries, given the same local rheological model for flow within the surface layer. It has been found both theoretically (19) and experimentally (22) that the hydrodynamic thickness is often much larger than the layer thickness determined optically by ellipsometry or by neutron scattering.

The effects of adsorbed polymers on the steady translation and steady rotation of a single spherical particle were determined by Anderson and Kim (21) using a method of matched asymptotic expansions to solve the Stokes flow equations within and outside the polymer layer. The results for the drag force and torque produced by the fluid on the particle, expressed as the hydrodynamic thickness of the adsorbed polymer layer, are accurate to $O\left(\lambda_{1}^{2}\right)$ where $\lambda_{1}$ is the ratio of the polymer-layer length scale to the particle radius. Their calculations indicated that (i) the $O\left(\lambda_{1}^{2}\right)$ term is negative, meaning the hydrodynamic thickness decreases as the particle radius decreases assuming all other conditions are constant, (ii) the free-draining assumption for flow through the 
polymer chains applies accurately for calculating the hydrodynamic thickness if the Stokes radius of the polymer segments is much smaller than the length scale of the polymer layer, and (iii) the presence of only a small amount of adsorbed polymer tails can make a significant contribution to the hydrodynamic thickness if the length scale of the tails exceeds the length scale of the loops by a factor of 2 or more.

In many practical situations, colloidal particles are not isolated and will move in the presence of neighboring particles and/or boundaries. Although the translational and rotational motions of a colloidal sphere coated with a layer of adsorbed polymers in an unbounded liquid were analyzed (21), the boundary effect on the movement of such particles has not yet been reported. The purpose of this work is to obtain insights into the boundary effects on the motion of a polymer-coated particle within a small pore. This type of problem is difficult to solve due to the structural difference for hydrodynamics within and outside the polymer layer and the complexity of the actual system geometry. In order to avoid the mathematical difficulties encountered in the sphere and cylindrical pore problem, we choose to study the translational and rotational motions of a spherical particle surrounded by an adsorbed polymer layer located at the center of a spherical cavity that may also bear a layer of adsorbed polymers on its inside wall. Although the geometry of spherical cavity is an idealized abstraction of any real system, the results obtained in this geometry have been shown to be in good agreement with available expressions for the boundary effects on the partition coefficient $(23,24)$, settling velocity $(25,26)$, and electrophoretic mobility $(27,28)$ of a "bare" particle in a cylindrical pore. Our analysis indicates that the boundary effects on the motion of a polymer-coated particle can be significant in general situations.

\section{TRANSLATION OF A PARTICLE IN A SPHERICAL CAVITY}

In this section we consider the quasisteady translational motion of a spherical particle of radius $R_{1}$ in a concentric spherical cavity (or pore) of radius $R_{2}$ filled with an incompressible Newtonian fluid. Both the surfaces of the particle and of the inside wall of the cavity are covered by a layer of adsorbed polymers. The thicknesses of the two surface polymer layers are characterized by length scales $\delta_{1}$ and $\delta_{2}$ (based on loops if both loops and tails are present), which are assumed to be small in comparison with $R_{1}$ and $R_{2}$, respectively, and with $R_{2}-R_{1}$. So, these two thin layers will not overlap with each other. In general, the length scale of a surface polymer layer depends on the molecular weight of the polymer, the amount of the polymer adsorbed, and the relative interactions among the polymer, interface, and fluid. The particle velocity equals $U e_{z}$, where ${\underset{\sim}{z}}_{z}$ is the unit vector in the positive $z$ (axial) direction. The Reynolds number is assumed to be small.

The fluid flow between the particle and the cavity is governed by the modified Stokes equations (21):

$$
\begin{gathered}
\nabla \cdot\left\{\mu\left[\nabla \underset{\sim}{\mathrm{y}}+(\nabla \underset{\sim}{\mathrm{y}})^{T}\right]\right\}-\nabla p-\zeta \rho f\left[\underline{\mathrm{v}}-\underline{\sim}^{(p)}\right]=0, \\
\nabla \cdot \underset{\sim}{\mathrm{v}}=0 .
\end{gathered}
$$

Here, $\mathrm{v}$ is the fluid velocity, $p$ is the hydrodynamic pressure, $\zeta$ is the friction coefficient of an isolated polymer segment, $\rho$ is the density of polymer segments at the position in question, $\mathrm{v}^{(p)}$ is the velocity of the segments, $f$ is a function of $\rho$ accounting for the hydrodynamic interactions among the segments, and $\mu$ is the local fluid viscosity which also varies with $\rho$. Previous studies $(1,2,17)$ established that the density of polymer segments in the loops decays exponentially with the distance from the solid surface and the segment density in the tails can exceed the value in the bulk solution much farther from the surface. For a free-draining model for flow through the segments $f=1$, while segment-segment interactions cause $f$ to increase with $\rho$. It is understood that $\mathbf{v}^{(p)}=$ $Q$ in the polymer layer adjacent to the stationary cavity wall and $\mathrm{v}^{(p)}=U e_{z}$ in the surface layer surrounding the translating particle.

Since the flow field is axially symmetric, it is convenient to introduce the Stokes stream function $\Psi$ which satisfies Eq. [2.1b] and is related to the velocity components in the spherical coordinate system $(r, \theta, \phi)$, with its origin at the particle center, by

$$
\mathrm{v}_{r}=-\frac{1}{r^{2} \sin \theta} \frac{\partial \Psi}{\partial \theta}, \quad \mathrm{v}_{\theta}=\frac{1}{r \sin \theta} \frac{\partial \Psi}{\partial r}
$$

Taking the curl of Eq. [2.1a] and applying Eqs. [2.1b] and [2.2] gives a fourth-order linear partial differential equation for $\Psi$ :

$$
\begin{aligned}
\mu E^{4} \Psi+ & 2 \frac{d \mu}{d r} \frac{\partial}{\partial r}\left(E^{2} \Psi\right)+2\left(r \frac{d^{2} \mu}{d r^{2}}-\frac{d \mu}{d r}\right) \frac{\partial}{\partial r}\left(\frac{1}{r} \frac{\partial \Psi}{\partial r}\right) \\
& -\frac{d^{2} \mu}{d r^{2}} E^{2} \Psi-\lambda_{i}^{-2}\left(\beta_{i} E^{2} \Psi+\frac{d \beta_{i}}{d r} \frac{\partial \Psi}{\partial r}\right)=0,
\end{aligned}
$$

where $i$ can be either 1 or 2 , the dimensionless parameters $\beta_{i}$ and $\lambda_{i}$ are defined as

$$
\beta_{i}=\frac{\delta_{i}^{2} \zeta \rho f}{\mu_{s}}, \quad \lambda_{i}=\frac{\delta_{i}}{R_{i}},
$$


and the axisymmetric Stokesian operator $E^{2}$ is given by

$$
E^{2}=\frac{\partial^{2}}{\partial r^{2}}+\frac{\sin \theta}{r^{2}} \frac{\partial}{\partial \theta}\left(\frac{1}{\sin \theta} \frac{\partial}{\partial \theta}\right) .
$$

In Eq. [2.3], dimensionless variables are used, with $r$ normalized by the radius $R_{i}, \mu$ by the solvent viscosity $\mu_{s}$, and $\Psi$ by $U R_{i}^{2}$. The parameter $\beta_{i}$ denotes the ratio of the frictional force exerted by the polymer segments on the fluid to the viscous force of the bulk fluid, and is $\mathrm{O}(1)$ with respect to $\lambda_{i}$ which has been assumed to be small. Equation [2.3] must be solved subject to the following boundary conditions:

$$
\begin{aligned}
& r=R_{1}: \underset{\sim}{\mathrm{y}}=U e_{z}, \\
& r=R_{2}: \underset{\mathrm{y}}{ }=0 .
\end{aligned}
$$

The drag force (in the $z$ direction) exerted by the fluid on the particle surface $r=R_{1}$ is (26)

$$
F_{d}=\pi \mu_{s} \int_{0}^{\pi} r^{3} \sin ^{3} \theta \frac{\partial}{\partial r}\left(\frac{E^{2} \Psi}{r^{2} \sin ^{2} \theta}\right) r d \theta .
$$

In terms of the equivalent hydrodynamic thickness $L$ of the adsorbed polymer layer surrounding the particle, the drag force is given by the Stokes law with a wall correction,

$$
F_{d}=-6 \pi \mu_{s}\left(R_{1}+L\right) U K
$$

For the system specified by Eq. [2.6], the wall correction factor $K=-4 D_{0} / 3 R_{1}$, where $D_{0}$ is defined by Eq. [2.12b]. The hydrodynamic thickness of the polymer layer can be expressed as

$$
L=A R_{1} \lambda_{1}\left(1+B \lambda_{1}\right)+O\left(\lambda_{1}^{3}\right)
$$

By combining Eqs. [2.7]-[2.9] after solving Eqs. [2.3] and [2.6] for $\Psi$, one can obtain dimensionless parameters $A$ and $B$. Note that $K \geqslant 1, A$ is positive, and $B$ represents the correction for the particle curvature.

Equation [2.3] poses a singular perturbation problem when $\lambda_{1} \ll 1$ and $\lambda_{2} \ll 1$. In the "outer region," where $r-R_{1} \gg$ $R_{1} \lambda_{1}$ and $R_{2}-r \gg R_{2} \lambda_{2}$, one has $\mu=\mu_{s}$ and $\beta_{i}=0$ and the solution can be written as

$$
\begin{aligned}
\Psi^{(o)} & =\left(C r^{-1}+D r+E r^{2}+F r^{4}\right) U \sin ^{2} \theta, \\
C & =C_{0}+\lambda_{1} C_{1}+\lambda_{1}^{2} C_{2}+\cdots \\
D & =D_{0}+\lambda_{1} D_{1}+\lambda_{1}^{2} D_{2}+\cdots \\
E & =E_{0}+\lambda_{1} E_{1}+\lambda_{1}^{2} E_{2}+\cdots \\
F & =F_{0}+\lambda_{1} F_{1}+\lambda_{1}^{2} F_{2}+\cdots
\end{aligned}
$$

The constants $C_{0}, D_{0}, E_{0}$, and $F_{0}$ can be obtained by solving for the corresponding motion of a "bare" spherical particle in a "bare" spherical cavity, with the result

$$
\begin{aligned}
& C_{0}=\frac{1}{4} R_{1}^{3}\left(1-l^{3}\right) \varpi, \\
& D_{0}=-\frac{3}{4} R_{1}\left(1-l^{5}\right) \varpi, \\
& E_{0}=\frac{1}{8}\left(9 l-5 l^{3}-4 l^{6}\right) \varpi, \\
& F_{0}=-\frac{3}{8} R_{1}^{-2}\left(l^{3}-l^{5}\right) \varpi,
\end{aligned}
$$

where

$$
l=\frac{R_{1}}{R_{2}}, \varpi=\left(1-\frac{9}{4} l+\frac{5}{2} l^{3}-\frac{9}{4} l^{5}+l^{6}\right)^{-1} .
$$

The unknown constants $C_{n}, D_{n}, E_{n}$, and $F_{n}$ for $n \geqslant 1$ are to be determined by matching $\Psi^{(O)}$ with the solution to Eq. [2.3] in the "inner regions." Combination of Eqs. [2.7][2.11] results in

$$
A=\frac{D_{1}}{D_{0}}, \quad B=\frac{D_{2}}{D_{1}} .
$$

Within the "inner region" adjacent to the particle, where $r-R_{1} \approx O\left(R_{1} \lambda_{1}\right)$, a solution to Eq. [2.3] with the reference frame moving with the particle is sought in the form

$$
\Psi_{i}^{(I)}=\left[\lambda_{i}^{2} F_{i 2}\left(y_{i}\right)+\lambda_{i}^{3} F_{i 3}\left(y_{i}\right)+\cdots\right] U \sin ^{2} \theta
$$

where $i=1$ and the variable $y_{1}=\lambda_{1}^{-1}\left(r-R_{1}\right)$. Within the "inner region" near the inside wall of the cavity, in which $R_{2}-r \approx O\left(R_{2} \lambda_{2}\right)$, the solution to Eq. [2.3] can also be written in the form of Eq. [2.15] with $i=2$ and the variable $y_{2}=\lambda_{2}^{-1}\left(R_{2}-r\right)$. Substituting Eq. [2.15] into Eqs. [2.3] and [2.6] and collecting terms of equal orders in $\lambda_{i}$ generates the following equations for $F_{\text {in }}\left(y_{i}\right)$ up to $n=3$ :

$$
\begin{array}{r}
\frac{R_{i}}{\mu_{s}} \frac{d}{d y_{i}}\left(\mu \frac{d^{2} F_{i 2}}{d y_{i}^{2}}\right)-\frac{\beta_{i}}{R_{i}} \frac{d F_{i 2}}{d y_{i}}=0 \\
y_{i}=0: F_{i 2}=\frac{d F_{i 2}}{d y_{i}}=0 \\
\frac{R_{i}}{\mu_{s}} \frac{d}{d y_{i}}\left(\mu \frac{d^{2} F_{i 3}}{d y_{i}^{2}}\right)-\frac{\beta_{i}}{R_{i}} \frac{d F_{i 3}}{d y_{i}} \\
=(3-2 i) \frac{2}{\mu_{s}} \frac{d \mu}{d y_{i}} \frac{d F_{i 2}}{d y_{i}}-c_{i}
\end{array}
$$




$$
y_{i}=0: F_{i 3}=\frac{d F_{i 3}}{d y_{i}}=0,
$$

where $i=1$ or 2 and $c_{i}$ are integration constants to be determined by matching the inner and outer solutions.

The unknown boundary conditions on $F_{\text {in }}$ at $y_{i} \rightarrow \infty$ and the unknown constants $C_{n}, D_{n}, E_{n}$, and $F_{n}$ can be obtained by matching Eqs. [2.10] and [2.15] at equivalent orders in $\lambda_{i}$ :

$$
\lim _{y_{1} \rightarrow \infty} \Psi_{1}^{(I)}=\left[\Psi^{(O)}+\frac{1}{2} U r^{2} \sin ^{2} \theta\right]_{r \rightarrow R_{1}+\lambda_{1} y_{1}}
$$

and

$$
\lim _{y_{2} \rightarrow \infty} \Psi_{2}^{(I)}=\left[\Psi^{(O)}\right]_{r \rightarrow R_{2}-\lambda_{2} y_{2}} .
$$

In Eq. [2.18a], the second term in brackets accounts for the difference in the reference frames used for $\Psi_{1}^{(I)}$ and $\Psi^{(O)}$. After completing this matching we obtain the following equations:

$$
\begin{aligned}
& 0=W_{i 0}+\left(1-\frac{i}{2}\right) R_{1}^{2}, \\
& 0=W_{i 1}+(3-2 i) y_{i}\left(\frac{\lambda_{i}}{\lambda_{1}}\right) X_{i 0}+(2-i) R_{1} y_{1},
\end{aligned}
$$

$$
\begin{aligned}
\lim _{y_{i} \rightarrow \infty}\left(\frac{\lambda_{i}}{\lambda_{1}}\right)^{2} F_{i 2}=W_{i 2} & +(3-2 i) y_{i}\left(\frac{\lambda_{i}}{\lambda_{1}}\right) X_{i 1} \\
& +y_{i}^{2}\left(\frac{\lambda_{i}}{\lambda_{1}}\right)^{2} Y_{i 0}+\left(1-\frac{i}{2}\right) y_{1}^{2},
\end{aligned}
$$

$$
\begin{aligned}
\lim _{y_{i} \rightarrow \infty}\left(\frac{\lambda_{i}}{\lambda_{1}}\right)^{3} F_{i 3} & =W_{i 3}+(3-2 i) y_{i}\left(\frac{\lambda_{i}}{\lambda_{1}}\right) X_{i 2} \\
& +y_{i}^{2}\left(\frac{\lambda_{i}}{\lambda_{1}}\right)^{2} Y_{i 1}+(3-2 i) y_{i}^{3}\left(\frac{\lambda_{i}}{\lambda_{1}}\right)^{3} Z_{i 0},
\end{aligned}
$$

where

$$
\begin{aligned}
W_{i k} & =R_{i}^{-1} C_{k}+R_{i} D_{k}+R_{i}^{2} E_{k}+R_{i}^{4} F_{k}, \\
X_{i k} & =-R_{i}^{-2} C_{k}+D_{k}+2 R_{i} E_{k}+4 R_{i}^{3} F_{k}, \\
Y_{i k} & =R_{i}^{-3} C_{k}+E_{k}+6 R_{i}^{2} F_{k}, \\
Z_{i k} & =-R_{i}^{-4} C_{k}+4 R_{i} F_{k},
\end{aligned}
$$

for $i=1$ or 2 and $k=0,1,2$, or 3 .
Equation [2.19a] and the derivative of Eq. [2.19b] with respect to $y_{i}$ are immediately satisfied with the solution of $C_{0}, D_{0}, E_{0}$, and $F_{0}$ given by Eq. [2.12]. The constants $C_{1}$, $D_{1}, E_{1}$, and $F_{1}$ can be determined by solving Eq. [2.19b] and the derivative of Eq. [2.19c] with the knowledge of $C_{0}, D_{0}$, $E_{0}$, and $F_{0}$, and then the constants $C_{2}, D_{2}, E_{2}$, and $F_{2}$ are to be determined by solving Eq. [2.19c] and the derivative of Eq. [2.19d]. The useful results are obtained in the following form:

$$
\begin{gathered}
D_{1}=-\frac{1}{3} \sum_{i=1}^{2} R_{i} b_{i}^{2}\left(\frac{\lambda_{i}}{\lambda_{1}}\right) \gamma_{i} \\
D_{2}=\frac{1}{3} \sum_{i=1}^{2}\left(\frac{\lambda_{i}}{\lambda_{1}}\right)^{2} \lim _{y_{i} \rightarrow \infty}\left[b_{i}\left(\frac{d F_{i 3}}{d y_{i}}+c_{i} \frac{y_{i}^{2}}{2 R_{i}}-d_{i} y_{i}\right)\right. \\
\left.+c_{i}\left(\frac{F_{i 2}}{R_{i}}-b_{i} \frac{y_{i}^{2}}{2 R_{i}}+b_{i} \gamma_{i} y_{i}\right)\right],
\end{gathered}
$$

where

$$
\gamma_{i}=\frac{1}{R_{i}} \lim _{y_{i} \rightarrow \infty}\left(-\frac{1}{b_{i}} \frac{d F_{i 2}}{d y_{i}}+y_{i}\right)
$$

and

$$
\begin{aligned}
b_{i}=\frac{3}{4} & (3-2 i)\left[2\left(\frac{R_{1}}{R_{i}}\right)^{6}-5 l^{3}+3 l^{5}\left(\frac{R_{1}}{R_{i}}\right)^{-4}\right] \varpi, \\
c_{i}=\frac{3}{2} & {\left[\left(\frac{R_{1}}{R_{i}}\right)^{6}+5 l^{3}-6 l^{5}\left(\frac{R_{1}}{R_{i}}\right)^{-4}\right] \varpi, } \\
d_{i}= & \frac{1}{2}(3-2 i)\left\{2 b _ { i } \gamma _ { i } \left[\left(\frac{R_{1}}{R_{i}}\right)^{6}-5 l^{3}+9 l^{5}\left(\frac{R_{1}}{R_{i}}\right)^{-4}\right.\right. \\
& \left.-5 l^{6}\left(\frac{R_{1}}{R_{i}}\right)^{-6}\right]-3\left(\frac{\lambda_{3-i}}{\lambda_{i}}\right) b_{3-i} \gamma_{3-i}\left[\left(\frac{R_{1}}{R_{i}}\right)^{6}\right. \\
& \left.\left.-5 l^{2}\left(\frac{R_{1}}{R_{i}}\right)^{2}+5 l^{3}-l^{5}\left(\frac{R_{1}}{R_{i}}\right)^{-4}\right]\right\} \varpi .
\end{aligned}
$$

Taking the differentiation of Eqs. [2.19c,d] with respect to $y_{i}$ twice, we obtain

$$
\lim _{y_{i} \rightarrow \infty} \frac{d^{2} F_{i 2}}{d y_{i}^{2}}=b_{i}
$$




$$
\lim _{y_{i} \rightarrow \infty} \frac{d^{2} F_{i 3}}{d y_{i}^{2}}=-c_{i} \frac{y_{i}}{R_{i}}+d_{i}
$$

Knowing that $\mu=\mu_{s}$ and $\beta_{i}=0$ as $y_{i} \rightarrow \infty$, one can find that Eqs. [2.16a] and [2.17a] are consistent with Eq. [2.24].

If we define new variables

$$
G_{i}=-\frac{1}{b_{i}} \frac{d F_{i 2}}{d y_{i}}, \quad H_{i}=-\frac{1}{c_{i}} \frac{d F_{i 3}}{d y_{i}}
$$

then Eqs. [2.16], [2.17], and [2.24] give

$$
\begin{gathered}
\frac{R_{i}}{\mu_{s}} \frac{d}{d y_{i}}\left(\mu \frac{d G_{i}}{d y_{i}}\right)-\frac{\beta_{i}}{R_{i}} G_{i}=0, \\
y_{i}=0: G_{i}=0, \\
y_{i} \rightarrow \infty: \frac{d G_{i}}{d y_{i}} \rightarrow-1
\end{gathered}
$$

$\frac{R_{i}}{\mu_{s}} \frac{d}{d y_{i}}\left(\mu \frac{d H_{i}}{d y_{i}}\right)-\frac{\beta_{i}}{\mu_{i}} H_{i}$

$$
\begin{gathered}
=1+2(3-2 i) \frac{b_{i}}{c_{i}} \frac{G_{i}}{\mu_{s}} \frac{d \mu}{d y_{i}}, \\
y_{i}=0: H_{i}=0, \\
y_{i} \rightarrow \infty: \frac{d H_{i}}{d y_{i}} \rightarrow \frac{y_{i}}{R_{i}}-\frac{d_{i}}{c_{i}} .
\end{gathered}
$$

The two parameters of Eq. [2.9] are obtained from Eqs. [2.14] and [2.21]:

$$
\begin{gathered}
A=-\frac{1}{3 D_{0}} \sum_{i=1}^{2} b_{i}^{2}\left(\frac{\lambda_{i}}{\lambda_{1}}\right) \lim _{y_{i} \rightarrow \infty}\left(G_{i}+y_{i}\right), \\
B=-\frac{1}{3 D_{0} A} \sum_{i=1}^{2} b_{i} c_{i}\left(\frac{\lambda_{i}}{\lambda_{1}}\right)^{2}\left[\lim _{y_{i} \rightarrow \infty}\left(H_{i}-\frac{y_{i}^{2}}{2 R_{i}}+\frac{d_{i}}{c_{i}} y_{i}\right)\right. \\
\left.+\frac{1}{R_{i}} \int_{0}^{\infty}\left(G_{i}+y_{i}-R_{i} \gamma_{i}\right) d y_{i}\right] .
\end{gathered}
$$

After the solutions of Eqs. [2.26] and [2.27] for given polymer segment density distributions $\rho\left(y_{i}\right)$ and the dependence of $\mu$ and $\beta_{i}$ on $\rho$ are obtained, parameters $A$ and $B$ can be evaluated from Eq. [2.28].

When there is no polymer adsorbed on the inside wall of the cavity, one has $\delta_{2}=\lambda_{2}=\Psi_{2}^{(I)}=0$. All of the variables with subscript $i=2$ (such as $y_{2}$ and $F_{2 n}$ ) then become trivial except for $R_{2}$, and the summation symbol in Eq. [2.28] should be replaced by setting $i=1$.

In the limit of $R_{2} / R_{1} \rightarrow \infty$, the boundary effect of the cavity on the motion of the particle disappears and all the variables with subscript $i=2$ are irrelevant to the hydrodynamic thickness of the polymer layer surrounding the particle. For this case, Eq. [2.23] gives $b_{1}=c_{1}=3 / 2$ and $d_{1}=(3 / 2) \gamma_{1}$, and Eq. [2.28] reduces to

$$
A_{\infty}=\gamma_{1}=\frac{1}{R_{1}} \lim _{y_{1} \rightarrow \infty}\left(G_{1}+y_{1}\right)
$$

$$
\begin{aligned}
B_{\infty}=\frac{1}{R_{1} \gamma_{1}} \lim _{y_{1} \rightarrow \infty} & \left(H_{1}-\frac{y_{1}^{2}}{2 R_{1}}+\gamma_{1} y_{1}\right) \\
& +\frac{1}{R_{1}^{2} \gamma_{1}} \int_{0}^{\infty}\left(G_{1}+y_{1}-R_{1} \gamma_{1}\right) d y_{1} .
\end{aligned}
$$

Here, we use the subscript " $\infty$ " to $A$ and $B$ to denote the limiting case of $R_{2} / R_{1} \rightarrow \infty$. The numerical values of $A_{\infty}$ and $B_{\infty}$ for various situations were given by Anderson and Kim (21). In general, $B_{\infty}$ for translation of a particle would be negative.

The drag force exerted on the particle written by Eq. [2.8] can also be expressed as

$$
F_{d}=-6 \pi \mu_{s}\left(R_{1}+L_{\infty}\right) U K\left[1+g \lambda_{1}+h \lambda_{1}^{2}+O\left(\lambda_{1}^{3}\right)\right],
$$

where

$$
L_{\infty}=A_{\infty} R_{1} \lambda_{1}\left(1+B_{\infty} \lambda_{1}\right)+O\left(\lambda_{1}^{3}\right) .
$$

Here, the hydrodynamic thickness of the polymer layer surrounding the particle is taken to be a constant equal to the value when the cavity is not present $\left(L_{\infty}\right)$, and the wall correction is given by an expansion in $\lambda_{1}$. A comparison between Eqs. [2.8] and [2.30] yields

$$
\begin{aligned}
& g=A-A_{\infty}, \\
& h=A B-A_{\infty} B_{\infty}-A_{\infty}\left(A-A_{\infty}\right) .
\end{aligned}
$$

\section{ROTATION OF A PARTICLE IN A SPHERICAL CAVITY}

We now consider the steady rotational motion of a spherical particle covered by a layer of adsorbed polymers situated at the center of a spherical cavity which is also coated with a polymer layer on its inside wall. The angular velocity of the particle is $\Omega e_{z}$. The fluid flow between the particle and the cavity is still governed by Eq. [2.1], and it must be solved subject to the following boundary conditions: 


$$
\begin{aligned}
& r=R_{1}: \underset{\sim}{\mathrm{v}}=\Omega r \sin \theta \underset{\sim}{e_{\phi}}, \\
& r=R_{2}: \underset{\mathrm{v}}{\mathrm{v}}=\underset{\sim}{0},
\end{aligned}
$$

where ${\underset{\sim}{\phi}}_{\phi}$ is the unit vector along changes in the azimuthal angle.

The effective hydrodynamic thickness $L$ of the adsorbed polymer layer surrounding the particle is now defined as the increase in radius of the particle needed to account for the torque $T_{d} e_{z}$ exerted on the particle by the fluid:

$$
T_{d}=-8 \pi \mu_{s}\left(R_{1}+L\right)^{3} \Omega K^{\prime}
$$

For the system specified by Eq. [3.1], the wall correction factor $K^{\prime}=M_{0} / R_{1}^{3}$ where $M_{0}$ is defined by Eq. [3.5a]. In the present case, the hydrodynamic thickness can also be expressed as Eq. [2.9]. Using Eqs. [3.2] and [2.9] after solving Eqs. [2.1] and [3.1] for the flow field, one can obtain parameters $A$ and $B$.

As in the previous section, the fluid is divided into an outer region (with $r-R_{1} \gg R_{1} \lambda_{1}$ and $R_{2}-r \gg R_{2} \lambda_{2}$ ) and two inner regions (with $r-R_{1} \approx O\left(R_{1} \lambda_{1}\right)$ and $R_{2}-r \approx$ $O\left(R_{2} \lambda_{2}\right)$ respectively). In the outer region, we can write the solution as

$$
\begin{aligned}
& p^{(O)}=\text { constant, } \\
& \underline{\sim}^{(O)}=\left(M r^{-2}+N r\right) \Omega \sin \theta{\underset{\sim}{\phi}}_{\phi}, \\
& M=M_{0}+\lambda_{1} M_{1}+\lambda_{1}^{2} M_{2}+\cdots, \\
& N=N_{0}+\lambda_{1} N_{1}+\lambda_{1}^{2} N_{2}+\cdots
\end{aligned}
$$

The constants $M_{0}$ and $N_{0}$ can be obtained easily by solving for the corresponding motion of a spherical particle in a spherical cavity in the absence of adsorbed polymer layers, with the result

$$
M_{0}=\frac{R_{1}^{3}}{1-l^{3}}, \quad N_{0}=-\frac{l^{3}}{1-l^{3}},
$$

where $l$ was defined by Eq. [2.13a]. The unknown constants $M_{n}$ and $N_{n}$ for $n \geqslant 1$ are to be determined by matching ${\underset{\sim}{\mathrm{V}}}^{(O)}$ with the solution to Eq. [2.1] in the inner regions. Combination of Eqs. [3.2]-[3.4] and [2.9] yields

$$
A=\frac{M_{1}}{3 M_{0}}, \quad B=\frac{M_{2}}{M_{1}}-\frac{M_{1}}{3 M_{0}}
$$

Within the inner region surrounding the particle, where $r$ $-R_{1} \approx O\left(R_{1} \lambda_{1}\right)$, a solution to Eq. [2.1] with the reference frame rotating with the particle is sought in the form

$$
\begin{aligned}
& p_{i}^{(I)}=\text { constant } \\
& {\underset{\mathfrak{y}}{i}}_{i}^{(I)}=\left[\lambda_{i} F_{i 1}^{\prime}\left(y_{i}\right)+\lambda_{i}^{2} F_{i 2}^{\prime}\left(y_{i}\right)+\cdots\right] \Omega \sin \theta \underset{e_{\phi}}{ },
\end{aligned}
$$

where $i=1$ and the variable $y_{1}=\lambda_{1}^{-1}\left(r-R_{1}\right)$. Within the inner region near the inside wall of the cavity, where $R_{2}-$ $r \approx O\left(R_{2} \lambda_{2}\right)$, the solution to Eq. [2.1] can also be expressed in the form of Eq. [3.7] with $i=2$ and the variable $y_{2}=$ $\lambda_{2}^{-1}\left(R_{2}-r\right)$. Substituting Eq. [3.7] into Eqs. [2.1] and [3.1] and collecting terms of equal orders in $\lambda_{i}$ produces the following equations for $F_{i n}^{\prime}\left(y_{i}\right)$ up to $n=2$ :

$$
\begin{gathered}
\frac{R_{i}}{\mu_{s}} \frac{d}{d y_{i}}\left(\mu \frac{d F_{i 1}^{\prime}}{d y_{i}}\right)-\frac{\beta_{i}}{R_{i}} F_{i 1}^{\prime}=0, \\
y_{i}=0: F_{i 1}^{\prime}=0
\end{gathered}
$$

$$
\begin{gathered}
\frac{R_{i}}{\mu_{s}} \frac{d}{d y_{i}}\left(\mu \frac{d F_{i 2}^{\prime}}{d y_{i}}\right)-\frac{\beta_{i}}{R_{i}} F_{i 2}^{\prime} \\
=(3-2 i) \frac{1}{\mu_{s}}\left(-2 \mu \frac{d F_{i 1}^{\prime}}{d y_{i}}+\frac{d \mu}{d y_{i}} F_{i 1}^{\prime}\right), \\
y_{i}=0: F_{i 2}^{\prime}=0 .
\end{gathered}
$$

The unknown boundary conditions on $F_{i n}^{\prime}$ at $y_{i} \rightarrow \infty$ and the unknown constants $M_{n}$ and $N_{n}$ can be obtained by matching Eqs. [3.3b] and [3.7b] at equivalent orders in $\lambda_{i}$ :

$$
\begin{aligned}
& \lim _{y_{1} \rightarrow \infty}{\underset{\sim}{1}}_{1}^{(I)}=\left[{\underset{\mathbf{v}}{(O)}}^{(O)}-\Omega r \sin \theta \underset{\sim}{e_{\phi}}\right]_{r \rightarrow R_{1}+\lambda_{1} y_{1}}, \\
& \lim _{y_{2} \rightarrow \infty}{\underset{\sim}{2}}_{2}^{(I)}=\left[{\underset{\sim}{\sim}}^{(O)}\right]_{r \rightarrow R_{2}-\lambda_{2} y_{2}} .
\end{aligned}
$$

The second term in brackets of Eq. [3.10a] accounts for the difference in the reference frames used for $\mathrm{v}_{1}^{(I)}$ and $\underline{v}^{(O)}$. After performing this matching one obtains

$$
0=W_{i 0}^{\prime}-(2-i) R_{1}
$$

$\lim _{y_{i} \rightarrow \infty}\left(\frac{\lambda_{i}}{\lambda_{1}}\right) F_{i 1}^{\prime}=W_{i 1}^{\prime}+(3-2 i) y_{i}\left(\frac{\lambda_{i}}{\lambda_{1}}\right) X_{i 0}^{\prime}-(2-i) y_{1}$,

$$
\lim _{y_{i} \rightarrow \infty}\left(\frac{\lambda_{i}}{\lambda_{1}}\right)^{2} F_{i 2}^{\prime}=W_{i 2}^{\prime}+(3-2 i) y_{i}\left(\frac{\lambda_{i}}{\lambda_{1}}\right) X_{i 1}^{\prime}+y_{i}^{2}\left(\frac{\lambda_{i}}{\lambda_{1}}\right)^{2} Y_{i 0}^{\prime}
$$


where

$$
\begin{aligned}
W_{i k}^{\prime} & =R_{i}^{-2} M_{k}+R_{i} N_{k}, \\
X_{i k}^{\prime} & =-2 R_{i}^{-3} M_{k}+N_{k}, \\
Y_{i k}^{\prime} & =3 R_{i}^{-4} M_{k},
\end{aligned}
$$

for $i=1$ or 2 and $k=0,1$, or 2 .

Solution of Eq. [3.11a] yields $M_{0}$ and $N_{0}$ given by Eq. [3.5]. The constants $M_{1}$ and $N_{1}$ can be obtained by solving Eq. [3.11b] using Eqs. [3.12] and [3.5], and then the constants $M_{2}$ and $N_{2}$ are determined by solving Eq. [3.11c]. The useful result is

$$
\begin{aligned}
& M_{1}=\frac{1}{3} \sum_{i=1}^{2} R_{i}^{3} b_{i}^{\prime 2}\left(\frac{\lambda_{i}}{\lambda_{1}}\right) \gamma_{i}^{\prime}, \\
& M_{2}=\frac{1}{3} \sum_{i=1}^{2} R_{i}^{2} b_{i}^{\prime}\left(\frac{\lambda_{i}}{\lambda_{1}}\right)^{2} \lim _{y_{i} \rightarrow \infty}\left(F_{i 2}^{\prime}-\frac{y_{i}^{2}}{2 R_{i}}+\frac{d_{i}^{\prime}}{c_{i}^{\prime}} y_{i}\right),
\end{aligned}
$$

where

$$
\gamma_{i}^{\prime}=\frac{1}{R_{i}} \lim _{y_{i} \rightarrow \infty}\left(\frac{F_{i 1}^{\prime}}{b_{i}^{\prime}}+y_{i}\right)
$$

and

$$
\begin{gathered}
b_{i}^{\prime}=3(3-2 i)\left(\frac{R_{1}}{R_{i}}\right)^{3} \frac{1}{1-l^{3}}, \\
c_{i}^{\prime}=6\left(\frac{R_{1}}{R_{i}}\right)^{3} \frac{1}{1-l^{3}}, \\
d_{i}^{\prime}=\left[l^{3}\left(\frac{R_{1}}{R_{i}}\right)^{-3} b_{i}^{\prime} \gamma_{i}^{\prime}+2\left(\frac{R_{1}}{R_{i}}\right)^{3} b_{i}^{\prime} \gamma_{i}^{\prime}\right. \\
\left.-3\left(\frac{\lambda_{3-i}}{\lambda_{i}}\right)\left(\frac{R_{1}}{R_{i}}\right)^{3} b_{3-i}^{\prime} \gamma_{3-i}^{\prime}\right] \frac{1}{1-l^{3}} .
\end{gathered}
$$

Taking the differentiation of Eq. [3.11] with respect to $y_{i}$, one has

$$
\begin{aligned}
& \lim _{y_{i} \rightarrow \infty} \frac{d F_{i 1}^{\prime}}{d y_{i}}=-b_{i}^{\prime}, \\
& \lim _{y_{i} \rightarrow \infty} \frac{d F_{i 2}^{\prime}}{d y_{i}}=c_{i}^{\prime} \frac{y_{i}}{R_{i}}-d_{i}^{\prime} .
\end{aligned}
$$

For given polymer segment density distributions $\rho\left(y_{i}\right)$ and the dependence of $\mu$ and $\beta_{i}$ on $\rho$, the parameters $A$ and $B$ of Eq. [2.9] can be obtained from Eqs. [3.6] and [3.13] after solving Eqs. [3.8], [3.9], and [3.16] for $F_{i 1}^{\prime}$ and $F_{i 2}^{\prime}$.

When there is no polymer attached to the cavity wall, one has $\mathrm{y}_{2}^{(I)}=0$. Then, all the variables with subscript $i=2$ are trivial except for $R_{2}$, and the summation symbol in Eq. [3.13] should be replaced by setting $i=1$.

In the limiting case of $R_{2} / R_{1} \rightarrow \infty$, Eq. [3.15] gives $b_{1}^{\prime}=$ 3, $c_{1}^{\prime}=6$, and $d_{1}^{\prime}=6 \gamma_{1}^{\prime}$; substitution of Eq. [3.13] into Eq. [3.6] results in

$$
\begin{gathered}
A_{\infty}=\gamma_{1}^{\prime}=\frac{1}{R_{1}} \lim _{y_{1} \rightarrow \infty}\left(\frac{1}{3} F_{11}^{\prime}+y_{1}\right), \\
B_{\infty}=\frac{2}{R_{1} \gamma_{1}^{\prime}} \lim _{y_{1} \rightarrow \infty}\left(\frac{1}{6} F_{12}^{\prime}-\frac{y_{1}^{2}}{2 R_{1}}+\gamma_{1}^{\prime} y_{1}\right)-\gamma_{1}^{\prime} .
\end{gathered}
$$

Again, the subscript " $\infty$ " to $A$ and $B$ is used to represent the unbounded case. It was found by Anderson and Kim (21) that $A_{\infty}$ is the same for translation and rotation of a particle and, if $\mu=\mu_{s}$ is assumed throughout the polymer layer, $B_{\infty}$ for rotation of a particle would be zero for any dependence of $\beta_{1}$ on $y_{1}$.

The torque exerted on the particle given by Eq. [3.2] can also be written as

$$
T_{d}=-8 \pi \mu_{s}\left(R_{1}+L_{\infty}\right)^{3} \Omega K^{\prime}\left[1+g \lambda_{1}+h \lambda_{1}^{2}+O\left(\lambda_{1}^{3}\right)\right],
$$

where $L_{\infty}$ is the hydrodynamic thickness of the polymer layer surrounding the particle in the absence of the cavity and can be expressed as Eq. [2.31]. By combining Eqs. [3.2], [3.18], [2.9], and [2.31], one obtains

$$
\begin{gathered}
g=3\left(A-A_{\infty}\right), \\
h=3\left(A^{2}+A B-A_{\infty}^{2}-A_{\infty} B_{\infty}\right)-9 A_{\infty}\left(A-A_{\infty}\right) .
\end{gathered}
$$

\section{RESULTS AND DISCUSSION}

The results for the translation and rotation of a colloidal sphere covered by a layer of adsorbed polymers in a concentric spherical cavity are presented in this section. For the case of the translational motion, the two hydrodynamic parameters $A$ and $B$ are calculated from Eq. [2.28] in which the variables $G_{i}$ and $H_{i}$ can be obtained by numerically solving Eqs. [2.26] and [2.27] in sequence. Similarly, for the case of the rotational motion, parameters $A$ and $B$ will be evaluated using Eqs. [3.6], [3.13], and [3.14] in which the variables $F_{i 1}^{\prime}$ and $F_{i 2}^{\prime}$ are to be determined by solving Eqs. [3.8], [3.9], and [3.16]. The only polymer-layer properties that are required are $\mu$ and $\beta_{i}$, which should be related to 
the segment density $\rho\left(y_{i}\right)$ by an appropriate hydrodynamic model. Previous calculations for an isolated sphere (21) have shown that the effects of $\mu>\mu_{s}$ are negligible compared to the effects of $\beta_{i}$. Therefore, we assume $\mu=\mu_{s}$ throughout the polymer layers in this and the following sections.

Following Anderson and Kim (21), we assume that the segment density has a form of two exponentially decaying distributions,

$$
\rho\left(y_{i}\right)=\rho_{i 0}\left(e^{-y_{i} / \delta_{i}}+\eta_{i} e^{-\alpha_{i} y_{i} / \delta_{i}}\right), \quad i=1 \text { or } 2,
$$

where the primary distribution $\left(\rho_{i 0} e^{-y_{i} / \delta_{i}}\right)$ represents segments in the loops and the secondary distribution denotes the tails. $\alpha_{i}$ is the ratio of loop-to-tail length scales for the relevant polymer layer and is smaller than unity. A theoretical model predicts that the value of $\alpha_{i}$ is about $0.5(17,29)$. It can be found from Eq. [4.1] that the fraction of segments contained in the tails is $\eta_{i} /\left(\alpha_{i}+\eta_{i}\right)$. Substituting Eq. [4.1] into Eq. [2.4a] yields

$$
\beta_{i}\left(y_{i}\right)=\beta_{i 0}\left(e^{-y_{i} / \delta_{i}}+\eta_{i} e^{-\alpha_{i} y_{i} / \delta_{i}}\right) f
$$

where

$$
\beta_{i 0}=\frac{\delta_{i}^{2} \zeta \rho_{i 0}}{\mu_{s}}=\frac{9}{2}\left(\frac{\delta_{i}}{a}\right)^{2} \phi_{i 0},
$$

$a\left(=\zeta / 6 \pi \mu_{s}\right)$ is the Stokes radius of each segment, and $\phi_{i 0}$ $\left(=4 \pi a^{3} \rho_{i 0} / 3\right)$ represents the volume fraction of segments based on the segment density at the surface of the particle or the cavity. For reasonable adsorption energies (i.e., not very close to the adsorption/desorption transition), $\phi_{i 0}$ is of order (but obviously below) unity. With the assumption that $\delta_{i} / a \gg 1$, the typical values of $\beta_{i 0}$ are expected to be in the range $10-1000$.

The hydrodynamic parameters $A$ and $B$ for the polymercoated particle in the cavity normalized by their values in the absence of the cavity $\left(A_{\infty}\right.$ and $\left.B_{\infty}\right)$ can be evaluated for a given distribution of $\beta_{i}$ as expressed by Eq. [4.2]. In our calculations, two cases of the spherical cavity have been considered: (i) there is no polymer adsorbed on the cavity wall $\left(\beta_{2}=0\right)$, and (ii) the polymer layer adjacent to the inside wall of the cavity has the same segment distribution as the layer surrounding the particle $\left(\beta_{2}=\beta_{1}\right)$. The RungeKutta-Fehlbery method (30) was employed to obtain the numerical solutions of the variables $G_{i}, H_{i}, F_{i 1}^{\prime}$, and $F_{i 2}^{\prime}$.

\subsection{Translation of a Particle in a Cavity}

For the quasisteady translation of a spherical particle situated at the center of a spherical cavity, results of $A / A_{\infty}$ and
$B / B_{\infty}$ for various values of $R_{1} / R_{2}$ are given in Table 1 for systems with no tails $\left(\eta_{i}=0\right)$ in the free-draining limit $(f$ $=1$ ). Three constant values, 10, 100, and 1000, are chosen for the parameter $\beta_{10}$, and the values of $A_{\infty}$ and $B_{\infty}$ for each case are also listed in this table. Note that, although $A_{\infty}$ is a function of $\beta_{10}$, the ratio $A / A_{\infty}$ is independent of this parameter. As expected, the presence of the cavity increases the value of $A$ and the ratio $A / A_{\infty}$ increases monotonically and rapidly with the increase of the ratio $R_{1} / R_{2}$. In the limit $R_{1} / R_{2}=0$, there is no boundary effect on the particle and $A$ $=A_{\infty}$. On the other hand, the ratio $B / B_{\infty}$ decreases monotonically from unity and soon becomes negative (or the value of $B$ increases monotonically from $B_{\infty}$, which is negative, and soon becomes positive) when the ratio $R_{1} / R_{2}$ increases from zero. Namely, for the general bounded case, the hydrodynamic thickness of the adsorbed polymer layer or the frictional drag on the particle increases as the particle radius decreases, assuming that the ratio $R_{1} / R_{2}$ and all other conditions are constant. Although the magnitude of $B_{\infty}$ decreases with the increase of $\beta_{10}$, the magnitude of $B$ or $B / B_{\infty}$ increases with the increase of $\beta_{10}$ for the general bounded case. In Table 1, it can also be observed that the cavity with an adsorbed polymer layer on its inside wall exerts more drag on the translating particle than does a bare cavity if all the other conditions are the same. Note that the boundary effect on the motion of the particle is very significant when the ratio $R_{1} / R_{2}$ approaches unity.

We next consider the effect of tails in the adsorbed polymer layer surrounding the particle when there is no polymer adsorbed on the cavity wall. Equations [2.26] and [2.27] are solved for $G_{1}$ and $H_{1}$ in the free-draining limit over a range of $\eta_{1}$ and $\alpha_{1}$. The results of parameter $B$ as a function of the ratio $R_{1} / R_{2}$ are plotted in Fig. 1 for typical cases of the fraction of polymer segments contained in the tails, $\eta_{1} /\left(\eta_{1}\right.$ $\left.+\alpha_{1}\right)$. The curve of parameter $A$ is not drawn since the ratio $A / A_{\infty}$ is not a function of $\eta_{1} /\left(\eta_{1}+\alpha_{1}\right)$ or $\alpha_{1}$ and its results were presented in Table 1. It is clear that the increase of the segment fraction in the tails or the increase of the relative length of the tails (with a decrease in $\alpha_{1}$ ) will increase the boundary effect on $B$ when all the other conditions are unchanged. This influence can be quite significant when the value of $\alpha_{1}$ is about (or less than) 0.5 . It implies that the interaction between the particle and the boundary in the presence of adsorbed polymers is to a large extent determined by long dangling tails.

We have also numerically solved for $G_{i}$ and $H_{i}$ for the system with no tails in the adsorbed polymer layers considering the hydrodynamic interactions among the polymer segments. In the calculations, we follow Anderson and Kim (21) and use a combination of a modified Brinkman equation at low $\phi_{i}$ and the Blake-Kozeny equation at high $\phi_{i}$ for the expression of function $f\left(\phi_{i}\right)$ : 
TABLE 1

Numerical R esults of $A / A_{\infty}$ and $B / B_{\infty}$ for a Translating Polymer-C oated Sphere Situated at the Center of a Spherical Cavity with Various Values of Parameters $R_{1} / R_{2}$ and $\beta_{10}$ for Systems with No Polymer Tails in the Free-Draining Limit

$-B / B_{\infty}$

\begin{tabular}{|c|c|c|c|c|c|c|c|c|}
\hline \multirow[b]{2}{*}{$R_{1} / R_{2}$} & \multicolumn{2}{|c|}{$A / A_{\infty}$} & \multicolumn{2}{|c|}{$\beta_{10}=10$} & \multicolumn{2}{|c|}{$\beta_{10}=100$} & \multicolumn{2}{|c|}{$\beta_{10}=1000$} \\
\hline & $\beta_{2}=0$ & $\beta_{2}=\beta_{1}$ & $\beta_{2}=0$ & $\beta_{2}=\beta_{1}$ & $\beta_{2}=0$ & $\beta_{2}=\beta_{1}$ & $\beta_{2}=0$ & $\beta_{2}=\beta_{1}$ \\
\hline 0 & 1.000 & 1.000 & -1.000 & -1.000 & -1.000 & -1.000 & -1.000 & -1.000 \\
\hline 0.1 & 1.280 & 1.283 & -0.017 & 0.074 & 1.740 & 1.995 & 4.375 & 4.879 \\
\hline 0.2 & 1.689 & 1.717 & 1.239 & 1.643 & 5.313 & 6.474 & 11.43 & 13.73 \\
\hline 0.3 & 2.266 & 2.380 & 2.733 & 3.763 & 9.702 & 12.70 & 20.16 & 26.10 \\
\hline 0.4 & 3.067 & 3.397 & 4.516 & 6.634 & 15.14 & 21.32 & 31.07 & 43.36 \\
\hline 0.5 & 4.192 & 4.990 & 6.751 & 10.69 & 22.20 & 33.68 & 45.37 & 68.17 \\
\hline 0.6 & 5.844 & 7.588 & 9.808 & 16.84 & 32.17 & 52.51 & 65.71 & 106.0 \\
\hline 0.7 & 8.511 & 12.19 & 14.57 & 27.17 & 48.07 & 84.19 & 98.34 & 169.7 \\
\hline 0.8 & 13.68 & 21.80 & 23.67 & 47.98 & 79.01 & 147.9 & 162.0 & 297.8 \\
\hline 0.9 & 28.85 & 51.40 & 50.29 & 110.6 & 170.4 & 339.5 & 350.6 & 682.9 \\
\hline 0.95 & 58.93 & 111.2 & 103.1 & 236.0 & 352.3 & 722.8 & 726.2 & 1453 \\
\hline \multirow[t]{2}{*}{0.99} & 299.0 & 591.0 & 524.9 & 1239 & 1806 & 3790 & 3728 & 7616 \\
\hline & & & \multicolumn{2}{|c|}{$\begin{array}{rr}A_{\infty}= & 3.4570 \\
B_{\infty} & =-0.9509\end{array}$} & \multicolumn{2}{|c|}{$\begin{aligned} A_{\infty} & =5.7596 \\
\mathrm{~B}_{\infty} & =-0.5712\end{aligned}$} & \multicolumn{2}{|c|}{$\begin{aligned} A_{\infty} & =8.0622 \\
B_{\infty} & =-0.4081\end{aligned}$} \\
\hline
\end{tabular}

$f=1+2.121 \phi_{i}^{1 / 2}+0.84 \phi_{i} \ln \phi_{i}+16.456 \phi_{i}$

for $\phi_{i}<0.29$,

[4.4a]

$$
f=\frac{8.34 \phi_{i}}{\left(1-\phi_{i}\right)^{3}} \text { for } \phi_{i}>0.29
$$

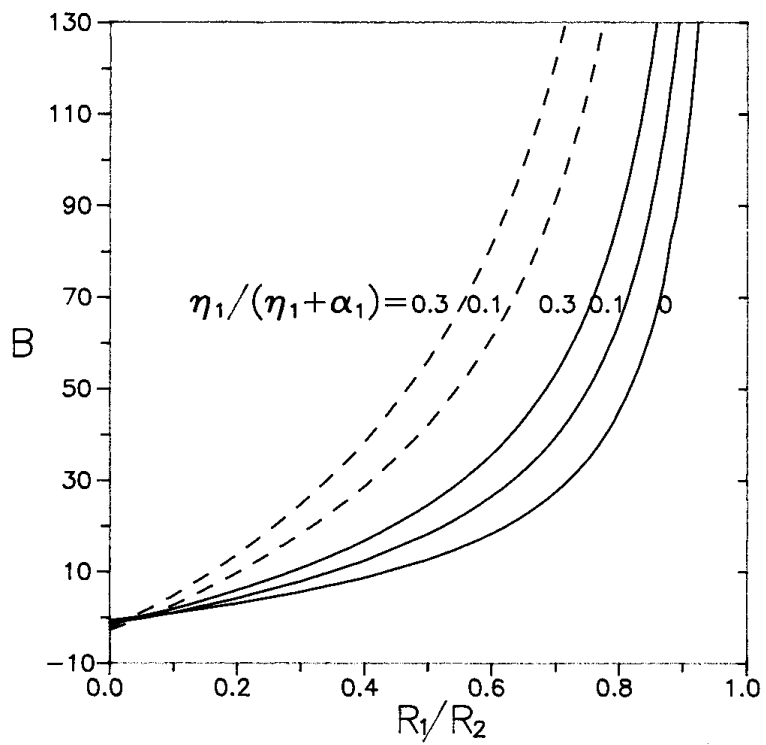

FIG. 1. The parameter $B$ for a translating polymer-coated sphere situated at the center of a bare spherical cavity as a function of $R_{1} / R_{2}$ with $\beta_{10}$ $=100$ in the free-draining limit $(f=1)$. The solid curves are plotted for the case of $\alpha=0.5$, and the dashed curves are plotted for the case of $\alpha=$ 0.25 . where $\phi_{i}=(4 / 3) \pi a^{3} \rho\left(y_{i}\right)$. The ratio $A / A_{\infty}$ is independent of $f\left(\phi_{i}\right)$ (in spite of the fact that $A_{\infty}$ is a weak function of $\delta_{1} /$ $a$ ) and its results are the same as those given in Table 1 . In Fig. 2, the results of parameter $B$ versus the ratio $R_{1} / R_{2}$ obtained using Eq. [4.4] for the case with a bare cavity are plotted for several values of $\beta_{10}$ and $\delta_{1} / a$. Although the

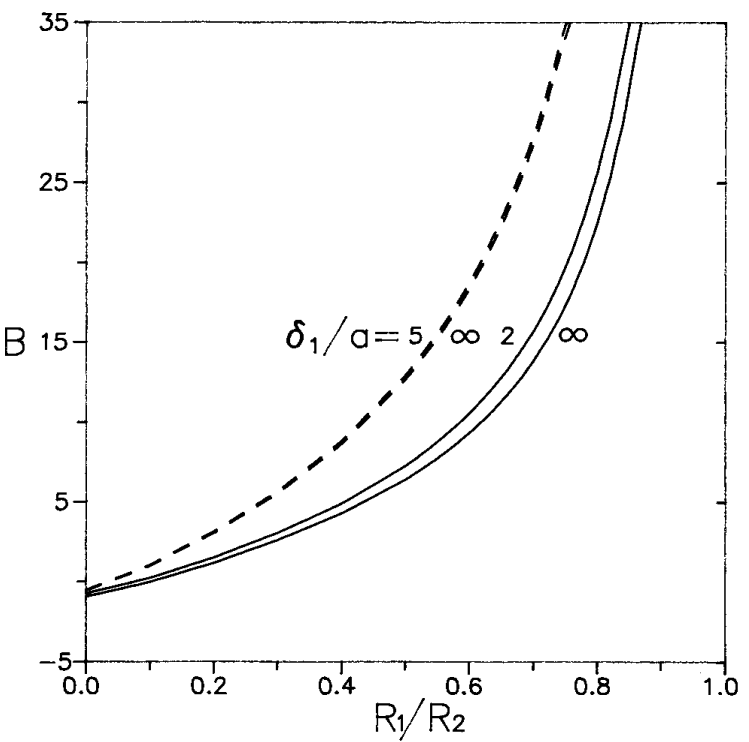

FIG. 2. The parameter $B$ for a translating polymer-coated sphere situated at the center of a bare spherical cavity as a function of $R_{1} / R_{2}$. The solid curves are plotted for the case of $\beta_{10}=10$, and the dashed curves are plotted for the case of $\beta_{10}=100$. Note that $\delta_{1} / a \rightarrow \infty$ represents the free-draining limit $(f=1)$. 
TABLE 2

N umerical Results of $A / A_{\infty}$ and $B$ for a Rotating Polymer-C oated Sphere Situated at the Center of a Spherical C avity with Various Values of Parameters $R_{1} / R_{2}$ and $\beta_{10}$ for Systems with No Polymer Tails in the Free-D raining Limit

$B$

\begin{tabular}{|c|c|c|c|c|c|c|c|c|}
\hline \multirow[b]{2}{*}{$R_{1} / R_{2}$} & \multicolumn{2}{|c|}{$A / A_{\infty}$} & \multicolumn{2}{|c|}{$\beta_{10}=10$} & \multicolumn{2}{|c|}{$\beta_{10}=100$} & \multicolumn{2}{|c|}{$\beta_{10}=1000$} \\
\hline & $\beta_{2}=0$ & $\beta_{2}=\beta_{1}$ & $\beta_{2}=0$ & $\beta_{2}=\beta_{1}$ & $\beta_{2}=0$ & $\beta_{2}=\beta_{1}$ & $\beta_{2}=0$ & $\beta_{2}=\beta_{1}$ \\
\hline 0 & 1.000 & 1.000 & 0.000 & 0.000 & 0.000 & 0.000 & 0.000 & 0.000 \\
\hline 0.1 & 1.001 & 1.001 & 0.007 & 0.008 & 0.012 & 0.014 & 0.016 & 0.020 \\
\hline 0.2 & 1.008 & 1.010 & 0.056 & 0.080 & 0.093 & 0.134 & 0.130 & 0.187 \\
\hline 0.3 & 1.028 & 1.036 & 0.192 & 0.321 & 0.320 & 0.536 & 0.447 & 0.750 \\
\hline 0.4 & 1.068 & 1.096 & 0.473 & 0.903 & 0.788 & 1.505 & 1.103 & 2.107 \\
\hline 0.5 & 1.143 & 1.214 & 0.988 & 2.092 & 1.646 & 3.485 & 2.303 & 4.878 \\
\hline 0.6 & 1.276 & 1.441 & 1.905 & 4.317 & 3.174 & 7.192 & 4.442 & 10.07 \\
\hline 0.7 & 1.522 & 1.888 & 3.610 & 8.412 & 6.014 & 14.02 & 8.418 & 19.62 \\
\hline 0.8 & 2.049 & 2.889 & 7.254 & 16.67 & 12.09 & 27.78 & 16.92 & 38.89 \\
\hline 0.9 & 3.690 & 6.111 & 18.60 & 40.54 & 30.99 & 67.55 & 43.38 & 94.55 \\
\hline 0.95 & 7.011 & 12.72 & 41.56 & 87.10 & 69.25 & 145.1 & 96.93 & 203.1 \\
\hline 0.99 & 33.67 & 66.01 & 225.9 & 456.2 & 376.3 & 760.1 & 526.8 & 1064 \\
\hline
\end{tabular}

boundary effect on $B$ is increased when the value of $\delta_{1} / a$ is decreased for a given value of $\beta_{10}$, the hydrodynamic interactions among the polymer segments produce relatively small effects on $B$.

\subsection{Rotation of a Particle in a Cavity}

For the steady rotational motion of a spherical particle in a concentric spherical cavity, results of $A / A_{\infty}$ and $B\left(B_{\infty}=\right.$ 0 in this situation) for various values of $\beta_{10}$ and $R_{1} / R_{2}$ are presented in Table 2 for systems with no tails in the freedraining limit. The value of $A_{\infty}$ for each case of $\beta_{10}$ is the same as that listed in Table 1 . It is understood that the data of $A / A_{\infty}$ in Table 2 are also valid for situations with the hydrodynamic interactions among the polymer segments. Similar to the case of translation of the particle, the ratio $A / A_{\infty}$ is independent of $\beta_{10}$ and it is a monotonic increasing function of $R_{1} / R_{2}$. The value of $B$ increases monotonically with the increase of $R_{1} / R_{2}$ or $\beta_{10}$. Also, the cavity with an adsorbed polymer layer on its inside wall exerts more drag on the rotating particle than does a bare cavity if all the other conditions are constant. A comparison between Tables 1 and 2 shows that the boundary effect on the rotation of a polymer-coated particle, while can be quite significant, is much weaker than the boundary effect on the translation of the particle.

To consider the effect of tails in the adsorbed polymer layer surrounding the particle, we have also solved Eqs. [3.8], [3.9], and [3.16] for $F_{11}^{\prime}$ and $F_{12}^{\prime}$ over a range of $\eta_{1}$ and $\alpha_{1}$ for the case of a bare cavity. The ratio $A / A_{\infty}$ is independent of the parameter $\eta_{1} /\left(\eta_{1}+\alpha_{1}\right)$ or $\alpha_{1}$. In Fig. 3, the results of parameter $B$ as a function of the ratio $R_{1} / R_{2}$ in the free-draining limit are plotted for typical values of $\eta_{1} /\left(\eta_{1}+\right.$ $\left.\alpha_{1}\right)$. Again, it is shown that the increase of the segment fraction in the tails or the relative length of the tails increases the boundary effect on parameter $B$.

We have also calculated parameter $B$ for various cases of rotation of a spherical particle in a spherical cavity considering the hydrodynamic interactions among the polymer segments. In general, the differences between these calculations and the free-draining results are rather small.

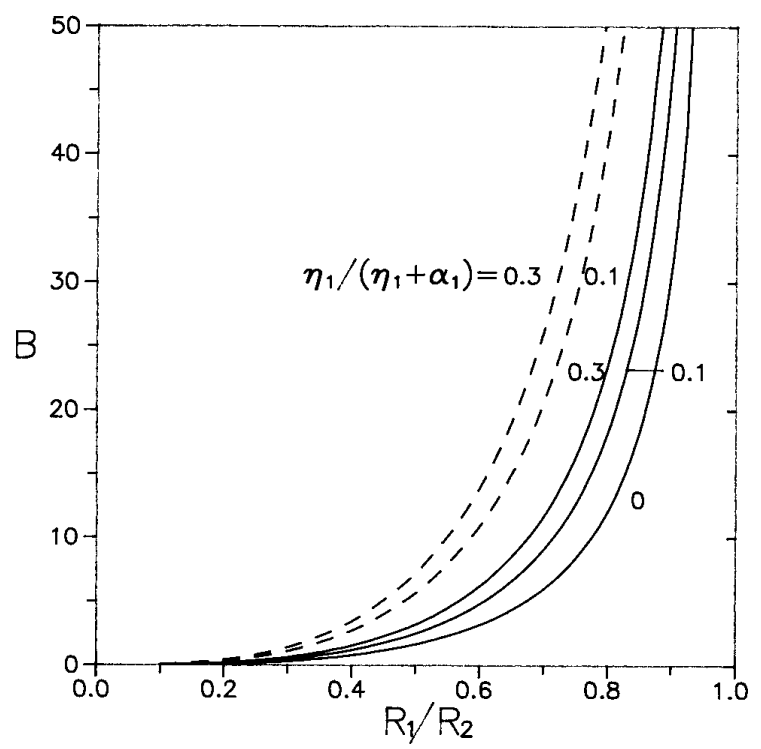

FIG. 3. The parameter $B$ for a rotating polymer-coated sphere situated at the center of a bare spherical cavity as a function of $R_{1} / R_{2}$ with $\beta_{10}=100$ in the free-draining limit $(f=1)$. The solid curves are plotted for the case of $\alpha$ $=0.5$, and the dashed curves are plotted for the case of $\alpha=0.25$. 


\section{CONCLUDING REMARKS}

The translational and rotational motion of a spherical particle coated with a layer of adsorbed polymers in a concentric spherical cavity has been analyzed in this work. The inside wall of the cavity may also be covered by an adsorbed polymer layer. The analysis provides simple equations which must be solved, given the polymer segment density distributions $\rho\left(y_{i}\right)$ and rheological parameters $\mu\left(y_{i}\right)$ and $\beta_{i}\left(y_{i}\right)$, to determine the parameters $A$ and $B$ of Eq. [2.9]. The hydrodynamic force and torque exerted on the particle, which is correct to $O\left(\lambda_{1}^{2}\right)$, can be calculated using Eqs. [2.8] and [3.2] and the results of $A$ and $B$. For the exponential polymer segment distribution given by Eq. [4.1], the ratio $A / A_{\infty}$ is found to be independent of the values of $\beta_{10}, \delta_{1} / a, \alpha_{1}$, and $\eta_{1} /\left(\eta_{1}+\alpha_{1}\right)$, and its results for various values of $R_{1} / R_{2}$ are listed in Tables 1 and 2. The dependence of $B$ on $\beta_{10}, \delta_{1} / a$, $\alpha_{1}, \eta_{1} /\left(\eta_{1}+\alpha_{1}\right)$, and $R_{1} / R_{2}$ is given in Tables 1 and 2 and Figs. 1-3. The results indicate that the boundary effects on the motion of a polymer-coated particle can be significant.

Throughout the calculations in the previous section we have assumed a simple exponential decay of the polymer segment density. This could be an oversimplification since the convex and concave nature of the surfaces of the particle and the cavity wall might adjust the excluded volume effects among the polymer segments. A segment density of the following form allows for curvature effects on polymer distribution:

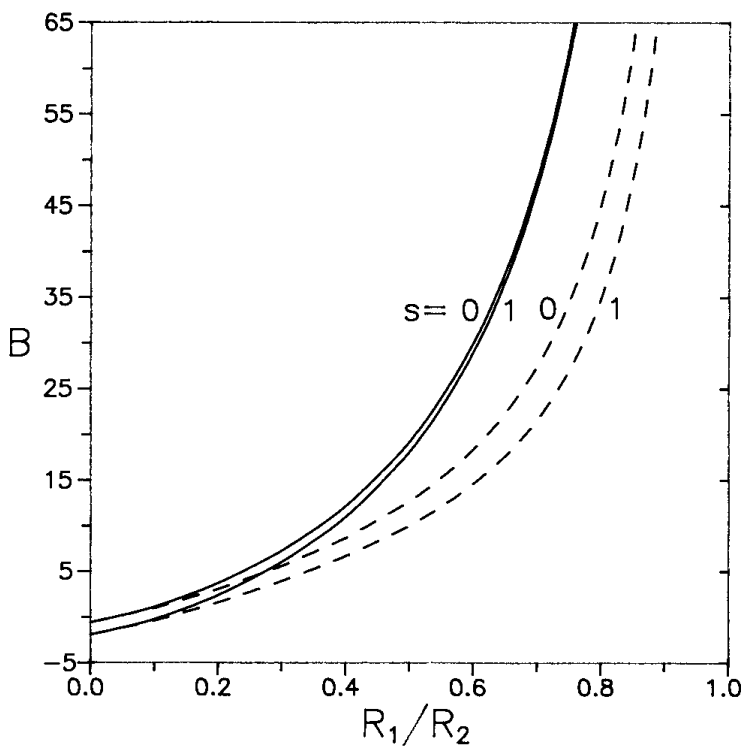

FIG. 4. The parameter $B$ for a translating polymer-coated sphere situated at the center of a spherical cavity as a function of $R_{1} / R_{2}$ with the segment density distributions given by Eq. [5.1] and $\beta_{10}=100$ in the freedraining limit. The dashed curves are plotted for the case of a bare cavity $\left(\beta_{2}=0\right)$, and the solid curves are plotted for the case of $\delta_{2}=\delta_{1}$ and $\beta_{20}$ $=\beta_{10}$.

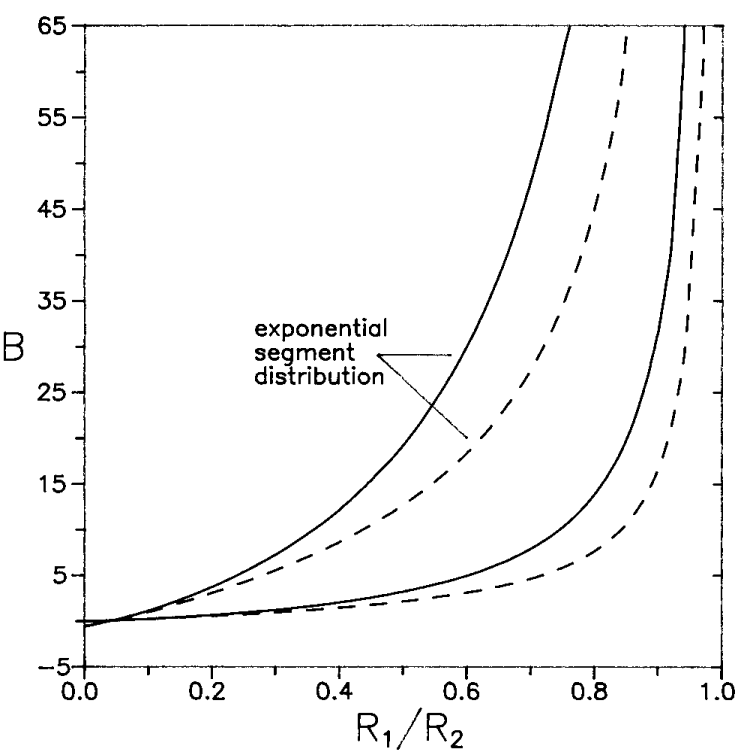

FIG. 5. The parameter $B$ for a translating polymer-coated sphere situated at the center of a spherical cavity as a function of $R_{1} / R_{2}$ with the segment density distributions given by Eq. [5.2] and $\beta_{10}=100$ in the freedraining limit. The dashed curves are plotted for the case of a bare cavity $\left(\beta_{2}=0\right)$, and the solid curves are plotted for the case of $\beta_{2}=\beta_{1}$. The corresponding results for the exponential segment distribution are also plotted for comparison.

$$
\rho\left(y_{i}\right)=\rho_{i 0} \frac{e^{-y_{i} / \delta_{i}}}{1+(3-2 i) s\left(y_{i} / R_{i}\right)} \quad i=1 \text { or } 2
$$

where $s$ should be a positive value. For systems with no tails in the free-draining limit, $\beta_{i}\left(y_{i}\right)$ has the form of the above equation with $\rho_{i 0}$ replaced by $\beta_{i 0}$. It is understood that parameters $A_{\infty}$ and $A$ are independent of the curvature coefficient $s$. We have numerically solved Eqs. [2.26] and [2.27] with the segment distribution given by Eq. [5.1] and substituted the solution of $G_{i}$ and $H_{i}$ into Eq. [2.28b] to compute parameter $B$. These calculations, which are plotted in Fig. 4 , indicate that the value of $B$ decreases with the increase of $s$ for various values of $R_{1} / R_{2}$ as one would expect.

One may wish to consider a polymer segment distribution that is uniform over a distance from the solid surfaces:

$$
\begin{array}{ll}
\rho\left(y_{i}\right)=\rho_{i 0} & \text { if } 0 \leqslant y_{i} \leqslant \delta_{i}, \\
\rho\left(y_{i}\right)=0 & \text { if } y_{i}>\delta_{i} .
\end{array}
$$

For this profile in the free-draining limit, $\beta_{i}\left(y_{i}\right)$ has the form of Eq. [5.2] with $\rho_{i 0}$ replaced by $\beta_{i 0}$ and it can be shown that

$$
\begin{aligned}
& A_{\infty}=1-\beta_{10}^{-1 / 2} \tanh \beta_{10}^{1 / 2}, \\
& B_{\infty}=-\frac{1}{A_{\infty} \beta_{10}}\left(1-\operatorname{sech} \beta_{10}^{1 / 2}\right)^{2} .
\end{aligned}
$$


Equation [5.3a] was obtained by Anderson and Kim (21), $R_{2}$ and it can be found that the value of $A_{\infty}$ is about six times $s$ greater (if $\beta_{10} \approx 100$ ) for an exponential distribution of $T_{d}$ segments than for the polymer uniformly distributed over a region of thickness $\delta_{1}$. Although $A_{\infty}$ is a function of $\beta_{10}$ for this segment distribution, the ratio $A / A_{\infty}$ is independent of the segment distribution and its results for the cases of $\beta_{2}$ $=0$ and $\beta_{2}=\beta_{1}$ have been given in Tables 1 and 2. In Fig. 5 , the numerical results of parameter $B$ for a translating polymer-coated sphere located at the center of a spherical cavity versus the ratio $R_{1} / R_{2}$ obtained using Eq. [5.2] for the segment distribution are plotted. The corresponding results for the case of an exponential distribution of segments are also plotted in the same figure for comparison. It can be seen that the boundary effect on $B$ is much weaker for the uniform distribution of segments over a distance from the solid surfaces than for the exponential distribution of segments.

\section{APPENDIX: NOMENCLATURE}

$a$

$A, B$

$A_{\infty}, B_{\infty}$

$b_{i}, c_{i}, d_{i}$

$b_{i}^{\prime}, c_{i}^{\prime}, d_{i}^{\prime}$

$C, D, E, F$

$C_{n}, D_{n}, E_{n}, F_{n}$

$e_{\phi}, e_{z}$

$f$

$F_{d}$

$F_{\text {in }}$

$F_{\text {in }}^{\prime}$

$G_{i}, H_{i}$

K

$K^{\prime}$

$l$

$L$

\section{$p$}

$M, N$

$M_{n}, N_{n}$

$R_{1}$
Stokes radius of a polymer segment (m)

parameters defined by Eq. [2.9]

parameters defined by Eq. [2.29] or [3.17]

coefficients defined by Eq. [2.23]

coefficients defined by Eq. [3.15]

coefficients defined by Eq. [2.10] $\left(\mathrm{m}^{3}, \mathrm{~m}\right.$, ,$- \mathrm{m}^{-2}$ )

coefficients defined by Eq. [2.11] or [2.12]

$$
\left(\mathrm{m}^{3}, \mathrm{~m},-, \mathrm{m}^{-2}\right)
$$

unit vectors in the $\phi$ and $z$ directions, respectively

parameter for the hydrodynamic interac-

tions among polymer segments defined by Eq. [2.1a]

hydrodynamic force exerted on the particle (N)

functions of $y_{i}$ defined by Eq. [2.15] $\left(\mathrm{m}^{2}\right)$

functions of $y_{i}$ defined by Eq. [3.7b] (m)

functions of $y_{i}$ defined by Eq. [2.25] (m)

wall correction factor defined by Eq. [2.8]

wall correction factor defined by Eq. [3.2]

$R_{1} / R_{2}$

effective hydrodynamic thickness of the polymer layer defined by Eq. [2.8] or [3.2] (m)

coefficients defined by Eq. [3.3b] $\left(\mathrm{m}^{3},-\right)$

coefficients defined by Eq. [3.4] or [3.5] $\left(\mathrm{m}^{3},-\right)$

hydrodynamic pressure $\left(\mathrm{N} / \mathrm{m}^{2}\right)$

radial spherical coordinate $(\mathrm{m})$

particle radius $(\mathrm{m})$ radius of the spherical cavity $(\mathrm{m})$

curvature coefficient defined by Eq. [5.1]

torque exerted by the fluid on the particle

$(\mathrm{N}$ m)

translational velocity of the particle $(\mathrm{m} / \mathrm{s})$

fluid velocity in the outer region $(\mathrm{m} / \mathrm{s})$

fluid velocities in the inner regions $(\mathrm{m} / \mathrm{s})$

velocity of polymer segments $(\mathrm{m} / \mathrm{s})$

$\lambda_{1}^{-1}\left(r-R_{1}\right)(\mathrm{m})$

$\lambda_{2}^{-1}\left(R_{2}-r\right)(\mathrm{m})$

axial coordinate $(\mathrm{m})$

ratio of loop-to-tail length scales for a poly-

mer layer

parameters defined by Eq. [2.4a]

parameters defined by Eq. [4.3]

coefficients defined by Eq. [2.22]

coefficients defined by Eq. [3.14]

length scale of the polymer layer surrounding the particle $(\mathrm{m})$

$\delta_{2}$

$\zeta$

$\eta_{i}$

$\theta, \phi$

$\lambda_{i}$

$\mu$

$\mu_{s}$

$\rho$

$\rho_{i 0}$

$\phi_{i}$

$\phi_{i 0}$

$\Psi$

$\Psi^{(O)}$

$\Psi_{i}^{(I)}$

๘

$\Omega$

\section{ACKNOWLEDGMENT}

This work was supported by the National Science Council of the Republic of China under Grant NSC 83-0402-E002-067.

\section{REFERENCES}

1. Silberberg, A., J. Phys. Chem. 66, 1872 (1962).

2. Hoeve, C. A. J., J. Chem. Phys. 44, 1505 (1966).

3. Dimarzio, E. A., and Rubin, R. J., J. Polym. Sci., Polym. Phys. Ed. 16, 457 (1978).

4. deGennes, P. G., Macromolecules 13, 1069 (1980).

5. Fuller, G. G., J. Polym. Sci., Polym. Phys. Ed. 21, 151 (1983).

6. Anderson, J. L., McKenzie, P. F., and Webber, R. M., Langmuir 7, 162 (1991). 
7. Stromberg, R. R., Tutas, D. J., and Passaglia, E., J. Phys. Chem. 69, 3955 (1965).

8. Doroszkowski, A., and Lambourne, R., J. Colloid Interface Sci. 26, 214 (1968).

9. Garvey, M. J., Tadros, Th. F., and Vincent, B., J. Colloid Interface Sci. 55, 440 (1976).

10. Kato, T., Nakamura, K., Kawaguchi, M., and Takahashi, A., Polym. J. 13, 1037 (1981).

11. Kim, J. T., and Anderson, J. L., Ind. Eng. Chem. Res. 30, 1008 (1991).

12. Carvalho, B. L., Tong, P., Huang, J. S., Witten, T. A., and Fetters, L. J., Macromolecules 26, 4632 (1993).

13. Napper, D. H., "Polymeric Stabilization of Colloidal Dispersions." Academic Press, London, 1983.

14. Russel, W. B., Saville, D. A., and Schowalter, W. R., “Colloidal Dispersions.' Cambridge University Press, London, 1989.

15. Gramain, Ph., and Myard, Ph., Macromolecules 14, 180 (1981).

16. McKenzie, P. F., Kapur, V., and Anderson, J. L., Colloids Surf. A 86, 263 (1994).

17. Scheutjens, J. M. H. M., and Fleer, G. J., J. Phys. Chem. 84, 178 (1980).
18. Kawaguchi, M., and Takahashi, A., Adv. Colloid Interface Sci. 37, 219 (1992).

19. Varoqui, R., and Dejardin, P., J. Chem. Phys. 66, 4395 (1977).

20. Hatano, A., Polymer 25, 1198 (1984).

21. Anderson, J. L., and Kim, J., J. Chem. Phys. 86, 5163 (1987).

22. Cohen Stuart, M. A., Waajen, F. H. W. H., Cosgrove, T., Vincent, B., and Crowley, T. L., Macromolecules 17, 1825 (1984).

23. Giddings, J. C., Kucera, E., Russell, C. P., and Myers, M. N., J. Phys. Chem. 72, 4397 (1968).

24. Glandt, E. D., AIChE J. 27, 51 (1981).

25. Bungay, P. M., and Brenner, H., Int. J. Multiphase Flow 1, 25 (1973).

26. Happel, J., and Brenner, H., "Low Reynolds Number Hydrodynamics.' Martinus Nijhoff, Dordrecht, The Netherlands, 1983.

27. Zydney, A. L., J. Colloid Interface Sci. 169, 476 (1995).

28. Keh, H. J., and Chiou, J. Y., AIChE J. 42, 1397 (1996).

29. Fleer, G. J., Cohen Stuart, M. A., Scheutjens, J. M. H. M., Cosgrove, T., and Vincent, B., "Polymers at Interfaces.' Chapman \& Hall, London, 1993.

30. Gerald, C. F., and Wheatley, P. O., "Applied Numerical Analysis," 5th ed. Addison-Wesley, Reading, MA 1994. 